\title{
Enhanced ceramides production by Lactobacillus rhamnosus IDCC 3201 and its proposed mechanism
}

\author{
Myun Soo Kim ${ }^{1 \dagger}$, Minjee Lee ${ }^{2 \dagger}$, Hanna Oh³, Wonsang Seo ${ }^{3}$, Gwang-Seob Kim², O-Hyun Ban ${ }^{2,4}$, Minhye Shin ${ }^{5}$, \\ Young Hoon Jung ${ }^{4,6^{*}}$ and Jungwoo Yang ${ }^{2^{*}}$ (D)
}

\begin{abstract}
The use of probiotics has been applied for a variety of fields (e.g., immune system, mental health, and heart). In this study, the feasibility of lysates from L. rhamnosus IDCC 3201 for cosmetic ingredients was evaluated. More specifically, enhanced ceramides production in human epidermal keratinocytes by the lysates and its proposed machanism were investigated through in vitro and genome analysis. In results, enhanced spingomyelinase activity and thereby increased ceramides production by the lysates from L. rhamnosus IDCC 3201 was observed. Furthermore, it was found that the existence of glucosylceramdase in L. rhamonsus IDCC 3201 was attributed to enhanced ceramides production. Finally, it was verified that the lysates from L. rhamonsus IDCC 3201 was regarded as safe for its use as cosmetic materials. Thus, these findings have significant implications that might lead to the development of functional and safe cosmetic products from probiotics.
\end{abstract}

Keywords: Ceramides, Glucosylceramidase, Probiotics, Skin health, Sphingomyelinase

\section{Introduction}

Recently, interest of skin health has been increased due to the escalating environmental stresses [1]. Especially, the most outer layer of the skin (stratum corneum) is thought to be a frotier of skin protection, and it is mainly composed of corneocytes and lipids [2]. These lipids mainly include several types of cholesterols, fatty acids, and ceramides [3]. Among them, ceramides, a member of sphingolipid family, play an important role in skin barrier function (e.g., skin homeostasis). Typically, ceramides are converted from glucosylceramides and sphingomyelins by glucosylceramidase (GCase) and sphingomyelinase (SMase), respectively [4]. Many clinical studies

\footnotetext{
*Correspondence: younghoonjung@knu.ac.kr; yjw@ildong.com ${ }^{\dagger}$ Myun Soo Kim and Minjee Lee equally contributed to this work ${ }^{2}$ Ildong Bioscience, 17 Poseunggongdan-ro, Pyeongtaek-si, Gyeonggi-do 17957, Republic of Korea

${ }^{4}$ School of Food Science and Biotechnology, Kyungpook National University, Daegu 41566, Republic of Korea

Full list of author information is available at the end of the article
}

showed that deficiency of ceramides is involved in atopic dermatitis (AD) and psoriasis directly or indirectly [5, 6]. Thus, one of the stategies for heathy skin should be to strengthen skin barrier through increased ceramides level [7]. For example, general approach is to regulate exogenous and endogenous factors involved in ceramides synthesis such as vitamin $\mathrm{C}$ and vitamin $\mathrm{D}[8,9]$.

Currently, probiotics have received considerable attention in cosmetic industry based on long-term uses of fermented broth for skin care $[10,11]$. Consistent with this notion, lysates of Streptococcus thermophilus S244 not only increased ceramides level of healthy elderly women but also alleviated symptoms of AD patients [12, 13]. In addition, Lactobacillus rhamnosus strain promoted epidermal barrier formation in human skin model [14]. However, there was no direct evidence which components of probiotics improve ceramides production in human skin and thereby skin barrier function.

In this study, enhanced ceramides production by lysates from L. rhamnosus IDCC 3201 and its machanism 
were investigated through in vitro and genome analysis. For this, firstly, the lysate from a variety of bacteria that exhibited the highest SMase activity was screened. Secondly, ceramides production by the screened probiotic strain was evaluated in human epidermal keratinocytes (HEK). Then, genome and metabolome analysis of the selected strain was perfomed to propose the mechanism. Finally, safety of the strain was assessed to determine whether it produces toxic compounds in human skin. Thus, this study contributes to the development of functional and safe cosmetic products from probiotics.

\section{Materials and methods}

\section{Bacterial strains and preparation of bacteral lysates}

L. rhamnosus IDCC 3201 from breast-fed infant's feces [15] and Streptococcus thermophilus IDCC 2201 from home-made yogurt [16] were industrial strains, while the other bacterial strains were isolated from kimchi (Korean fermented food). All the strains were incubated in an in-house medium designed by Ildong Bioscience, centrifuged, and freeze-dried to obtain cell density at $>10^{10}$ colony forming units (CFU)/g. Then, lyophilized strains were suspended in $500 \mathrm{~mL}$ of phosphate buffered solution (PBS) and was lysised physically by using a microfluidizer (PicoMax MN400BF; Micronox, Seongnam, Korea) to preprare bacterial lysates.

\section{Sphingomyelinase (SMase) activity}

SMase activity was measured using a SMase assay kit (Abcam; \# ab138876, Cambridge, UK). The principle of this assay kit was to quantify phosphocholine produced by the hydrolysis of sphingomyelin using colorimetric analysis at $655 \mathrm{~nm}$. Briefly, $50 \mu \mathrm{L}$ of sphingomyelin was incubated with lysates from five bacterial strains at $37^{\circ} \mathrm{C}$ for $1 \mathrm{~h}$. Then, color changes were detected using a microplate reader (SpectraMax iD3; Molecular Devices, CA, USA). Then, SMase activity was calculated according to calibration curves with the absorbance values of known amounts of SMase standards.

\section{Cell culture}

Normal human epidermal keratinocyte (HEK) cells were purchased from PromoCell (Heidelberg, Germany). Cells were grown in growth medium 2, supplemented with bovine pituitary extract, epidermal growth factor, Insulin, hydrocortisone, epinephrine, transferin and $0.06 \mathrm{mM}$ of $\mathrm{CaCl}_{2}$ (PromoCell). After confluence for 3-5 days at $37{ }^{\circ} \mathrm{C}$ with $5 \% \mathrm{CO}_{2}$, cells were maintained by sub-culturing the detached cells with a $0.25 \%$ trypsin-EDTA solution.

\section{Cell viability assay}

Cell viability of the HEK cells by tested bacterial lysates was measured using 3-(4,5-dimethylthiazol-2-yl)-2,5-diphenyltetrazolium bromide (MTT). Briefly, the HEK cells were cultured in 96-well plate and treated with the lysates of $L$. rhamnosus IDCC 3201 at cell densities ranging from $1 \times 10^{7}$ to $5 \times 10^{8} \mathrm{CFU} / \mathrm{mL}$ for $24 \mathrm{~h}$. Then, MTT was added to each 96 -well and incubated at $37^{\circ} \mathrm{C}$ for $90 \mathrm{~min}$. After removal of MTT, $100 \mu \mathrm{L}$ of dimethyl sulfoxide (DMSO) was added to dissolve formazan. The developed color was measured at $570-630 \mathrm{~nm}$ using a microplate reader (SpectraMax iD3).

\section{Ceramides production}

Ceramide contents in the suspension from the HEK cells were measured using an ELISA kit (Human Ceramides ELISA Kit; MyBioSource, CA). Briefly, The HEK cell culture suspension with SMase was treated to a 96-well plate which coated by human monoclonal antibody. The plate was incubated with biotin-labeled polyclonal antibody which is conjugated with avidin-peroxidase. Next, a chromogen solution was added into the plate for ELISA colormetric detection at $450 \mathrm{~nm}$. Finally, amounts of ceramide were calculated according to caribration curve prepared with ceramides standards.

\section{Real-Time PCR}

Gene expression for ceramides synthesis was investigated using real-time PCR. Total RNAs were isolated from HEK cells treated with either SMase (positive control) and bacterial lysates by a Trizol method. PCR reactions were performed using CFX Connect Real-Time PCR Detection System (Bio-Rad, Hercules, CA). The housekeeping gene, GAPDH was used as a constitutive control for normalization. The specific primers used for real-time PCR are listed in Table 1.

Table 1 Sequence of the primers used in the study

\begin{tabular}{lll}
\hline Gene & Strand & Sequence $\left(\mathbf{5}^{\prime}\right.$ - $\mathbf{3}^{\prime}$ ) \\
\hline SMPD3 & Forward & ACATCGATTCTCCCACCAACACCT \\
& Reverse & AATTCGCACAATGCAGCTGTCCTC \\
CERS1 & Forward & ACGCTACGCTATACATGGACAC \\
& Reverse & AGGAGGAGACGATGAGGATGAG \\
CERS2 & Forward & CCGATTACCTGCTGGAGTCAG \\
& Reverse & GGCGAAGACGATGAAGATGTTG \\
GAPDH & Forward & ACGGACTTCCTCGGTATAC \\
& Reverse & CGGTGACTGTAGCCATATTCG \\
\hline
\end{tabular}




\section{Bacterial gene search for ceramides biosynthesis}

The whole-genome sequencing of $L$. rhamnosus IDCC 3201 was performed by a PacBio RSII instrument with an Illumina platform (Macrogen, Seoul, Korea). A sequence of nucleotides was generated by single molecule realtime (SMRT) sequencing system. Contigs were constructed by pre-assembling seed reads, by generating a consensus sequence of the mapped reads, and by correcting and filtering the reads. Finally, a consensus sequence with higher quality was obtained after error-correction of the constructed contigs by Pilon (version 1.21).

To find bacterial enzymes involved in ceramides biosynthesis from probiotics, amino acid sequence of enzymes were collected: sphingomyelinase (EC 3.1.4.12); glucosylceramidase (EC 3.2.1.45); galactosylceramidase (EC 3.2.1.46), ceramides synthase (EC 2.3.1.24), and sphingolipid-4-desaturase (EC 1.14.19.17) [17] from UniProtKB database, and built hidden Markov models (HMMs), representing the conserved amino acid sequence patterns in these enzymes (Table 2). Then, genome of L. rhamnosus IDCC 3201 was targeted to search using the HMMsearch tool in HMMER package with the constructed profile of HMMs [18]. Finally, the candidate enzymes were verified using BLASTP tool in NCBI BLAST and HMMscan tool in HMMER package against SWISS PROT database and PFAM database, respectively.

\section{Metabolites analysis}

Bacterial lysates were diluted in ice-cold methanol to a final concentration of $80 \%$ with a vortex for $1 \mathrm{~min}$ on ice. After centrifugation at $13,000 \mathrm{~g}$ for $10 \mathrm{~min}$ at $4{ }^{\circ} \mathrm{C}$, the upper layer of the supernatant was collected, concentrated to dryness in a vacuum concentrator, and stored at $-80{ }^{\circ} \mathrm{C}$ prior to derivatization and analysis by GC-MS.
The extract was derivatized with $30 \mu \mathrm{L}$ of a solution of $20 \mathrm{mg} / \mathrm{mL}$ methoxyamine hydrochloride in pyridine (Sigma, St. Louis, MO) at $30{ }^{\circ} \mathrm{C}$ for $90 \mathrm{~min}$, and $50 \mu \mathrm{L}$ of N,O-Bis(trimethylsilyl)trifluoroacetamide (BSTFA; Sigma) was subsequently added at $60{ }^{\circ} \mathrm{C}$ for $30 \mathrm{~min}$. A mixture of fatty acid methyl esters and fluoranthene was added to the extract as internal standards. The GC-MS analysis was conducted using a Thermo Trace 1310 GC (Waltham, MA) coupled to a Thermo ISQ LT single quadrupole mass spectrometer (Waltham, MA). A DB5MS column with $60-\mathrm{m}$ length, $0.2-\mathrm{mm}$ i.d. and $0.25-\mu \mathrm{m}$ film thickness (Agilent, Santa Clara, CA) was used for separation. For analysis, the sample was injected at $300{ }^{\circ} \mathrm{C}$ and split ratio $1: 5$ with $7.5 \mathrm{~mL} / \mathrm{min}$ helium split flow. The metabolites were separated with $1.5 \mathrm{~mL}$ constant flow helium with an oven ramp of $50{ }^{\circ} \mathrm{C}(2 \mathrm{~min}$ hold $)$ to $180^{\circ} \mathrm{C}$ (8 min hold) at $5{ }^{\circ} \mathrm{C} / \mathrm{min}$, to $210^{\circ} \mathrm{C}$ at $2.5^{\circ} \mathrm{C} / \mathrm{min}$, and to $325^{\circ} \mathrm{C}(10 \mathrm{~min}$ hold $)$ at $5^{\circ} \mathrm{C} / \mathrm{min}$. The mass spectra were acquired in a scan range of $35-650 \mathrm{~m} / \mathrm{z}$ at an acquisition rate of 5 spectra per sec. The ionization mode was subjected to electron impact, and the temperature for the ion source was set to $270{ }^{\circ} \mathrm{C}$. The spectra were processed by Thermo Xcalibur software using automated peak detection, and the metabolites were identified by matching the mass spectra and retention indices of the NIST Mass spectral search program (version 2.0, Gaithersburg, MD). The metabolite data were then normalized based on the intensity of the fluoranthene internal standard.

\section{Safety evaluation of Lactobacillus rhamnosus IDCC 3201 Genome analysis}

The VFDB database was searched for virulence genes [19], and ResFider software (ver. 3.2) with the CARD database was searched for antibiotic resistance genes [20]. The search parameters were set to the identity

Table 2 Information of ceramide biosynthesis genes

\begin{tabular}{llll}
\hline Gene & Precursor & Enzyme & PFAM domain \\
\hline degs & Dihydroceramide & Sphingolipid 4-desaturase & PF00487 (FA_desaturase) \\
galc & Galactosylceramide & Galactosylceramidase & PF02057 (Glyco_hydro_59) \\
gba & Glucosylceramide & Glucosylceramidase & PF02055 (Glyco_hydro_30) \\
gba2 & Glucosylceramide & Non-lysosomal glucosylceramidase & PF12215 (Glyco_hydro_116N) \\
sph & Sphingomyelin & Sphingomyelin phosphodiesterase & PF03372 (Exo_endo_phos) \\
smpd1 & Sphingomyelin & Sphingomyelin phosphodiesterase & PF00149 (Metallophos) \\
smpd2 & Sphingomyelin & Sphingomyelin phosphodiesterase & PF03372 (Exo_endo_phos) \\
smpd3 & Sphingomyelin & Sphingomyelin phosphodiesterase & PF03372 (Exo_endo_phos) \\
smpd4 & Sphingomyelin & Sphingomyelin phosphodiesterase & PF14724 (mit_SMPDase) \\
enpp7 & Sphingomyelin & Sphingomyelin phosphodiesterase & PF01663 (Phosphodiest) \\
cers1 & Sphingosine & Sphingoid base N-stearoyltransferase & PF03798 (TRAM_LAG1_CLN8) \\
cers2 & Sphingosine & Very-long-chain ceramide synthase & PF03798 (TRAM_LAG1_CLN8) \\
cers3 & Sphingosine & Very-long-chain ceramide synthase & PF03798 (TRAM_LAG1_CLN8) \\
\hline
\end{tabular}


of $>80 \%$ and coverage of $>80 \%$ for gene identification. Transposases and transferases were annotated using the protein-protein basic local search program (BLASTP) against the NCBI GenBank proteins. Prophage regions were identified using PHASTER web-based program [21].

\section{In vitro analysis}

Lactobacillus rhamnosus IDCC 3201 was evaluated for susceptibility to antimicrobials including ampicillin, vancomycin, gentamicin, kanamycin, streptomycin, erythromycin, clindamycin, tetracycline, and chloramphenicol (Sigma-Aldrich, St. Louis, MO), which are typically used to treat enterococcal infections [22]. Briefly, a single colony from plate was inoculated to MRS broth and preincubated for $18 \mathrm{~h}$. The cultured cells and antibiotic solution were mixed in 96-well plate to obtain the cell density of $5 \times 10^{5} \mathrm{CFU} / \mathrm{mL}$, and the plate was then incubated at $37{ }^{\circ} \mathrm{C}$ anaerobically for $18 \mathrm{~h}$. The optical density was observed using a microplate reader (BioTek, Winooski, $\mathrm{VT}$ ). The minimal inhibitory concentrations (MICs) were determined compared to the cut-off values suggested by European Food Safety Authority (EFSA) [23].

\section{Results and discussion}

Screening of lactic acid bacteria with higher sphingomyelinase (SMase) acitivity

SMase is the key enzyme of sphingomyelin catabolism, converting into ceramides and phosphorylcholine [24]. Thus, decreased sphingomyelinase activity often leads to decreased ceramides level, resulting in skin troubles (e.g., atopic dermatitis) [25]. Here, various lactic acid bacteria were screened to obtain the strain(s), producing higher concentration of ceramides. For this, lysates from various lactic acid bacteria were prepared to observe whether the SMase increased. In particular, the lysates of tested strains used in this experiments were prepared from cell desity at $5 \times 10^{7} \mathrm{CFU} / \mathrm{mL}$. As a result, $L$. rhamnosus IDCC 3201 exhibited highest SMase activity $1.85 \pm 0.03 \mathrm{mU} / \mathrm{mL}$, while the other strains exhibited only at $0.28-0.43 \mathrm{mU} / \mathrm{mL}$ (Fig. 1a). The value of $L$. rhamnosus IDCC 3201 was 3.4 times higher than those of other strains, such as $L$. mesenteroides, L. plantarum, L. casei, and S. thermophilus. Consequently, L. rhamnosus IDCC 3201 strain was selected as the best performer with regard to SMase activity.

Next, optimal cell density of $L$. rhamnosus IDCC 3201 was evaluated to determine economically feasible concentration. The lysates from $1 \times 10^{7}$ to $5 \times 10^{10} \mathrm{CFU} / \mathrm{mL}$ were loaded onto the substractes. In results, SMase activity was observed to increase up to $1.65 \pm 0.09 \mathrm{mU} / \mathrm{mL}$, as cell density increased (Fig. 1b). In contrast, in the view of efficiency of enzymatic activity (enzymatic unit/cell density), when cell density was the lowest as $1 \times 10^{7} \mathrm{CFU}$, enzymatic efficiency of SMase was highest (Fig. 1b). Thus, cell amount of $1 \times 10^{7} \mathrm{CFU} / \mathrm{mL}$ of L. rahmonsus IDCC 3201, showing the highest SMase efficiency was selected as optimal cell density for further study.

\section{Enhanced ceramides production by L. rhamnosus IDCC 3201}

Ceramides, the major lipid consitituents in the upper epidermal layer play a role in maintaining skin barrier integrity [26]. Thus, this layer prevents the skin from excess water loss and protects skin against various stimuli, including bacterial or microbial infection [8].
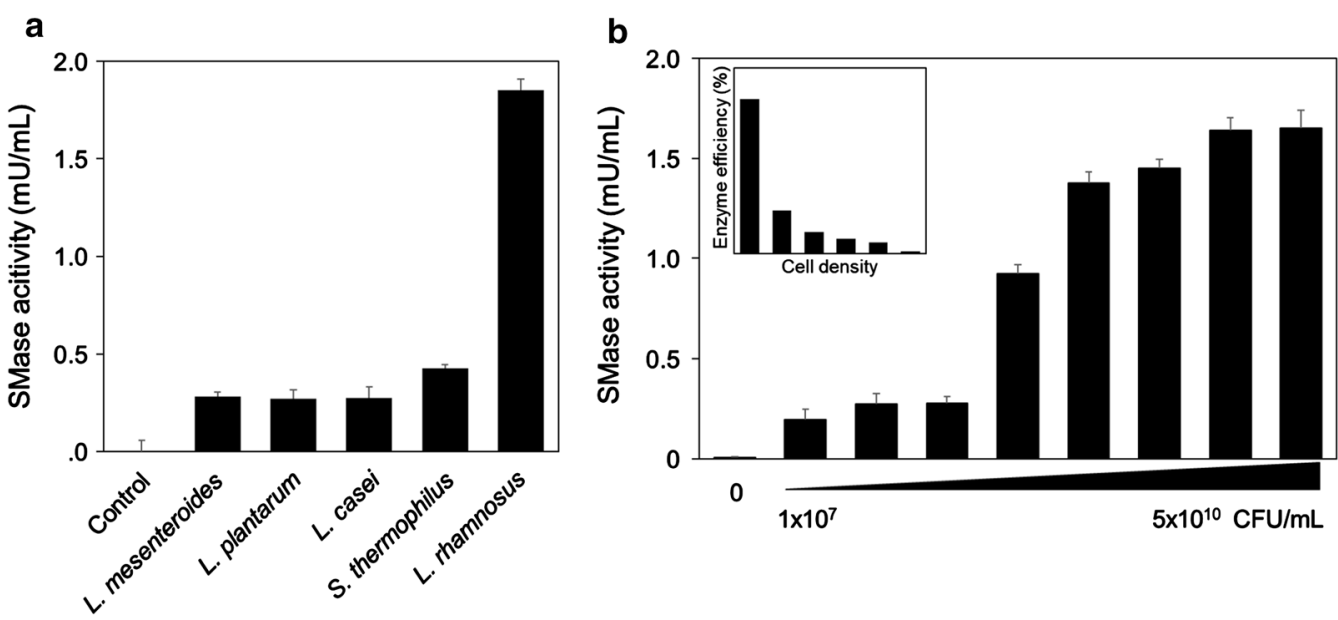

Fig. 1 Enhanced sphingomyelinase (SMase) activity by the lysates from L. rhamnosus IDCC 3201. a SMase activities of a variety of lactic acid bacteria. b Cell density-dependent SMase activity from L. rhamnosus IDCC 3201. The efficiency of enzymatic activity is expressed as enzymatic activity divided by cell density. The data represent means \pm standard deviations from triplicate experiments 


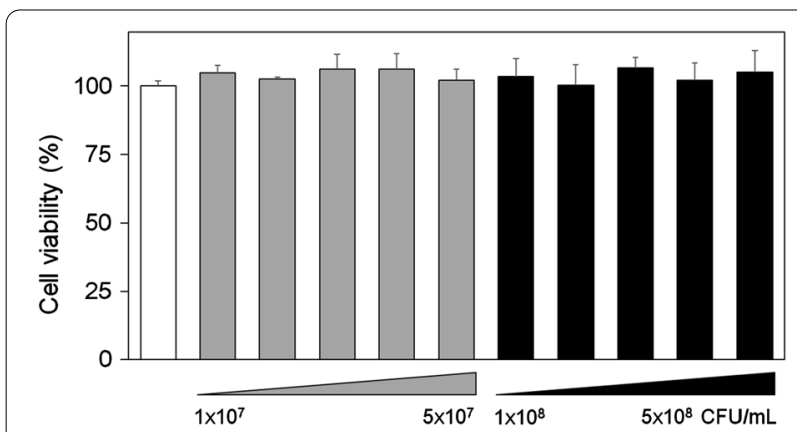

Fig. 2 Cytotoxic effect of L. rhamnosus IDCC 3201 on keratinocytes. Cell viability was measured by MTT-assay. The data represent means \pm standard deviations from triplicate experiments

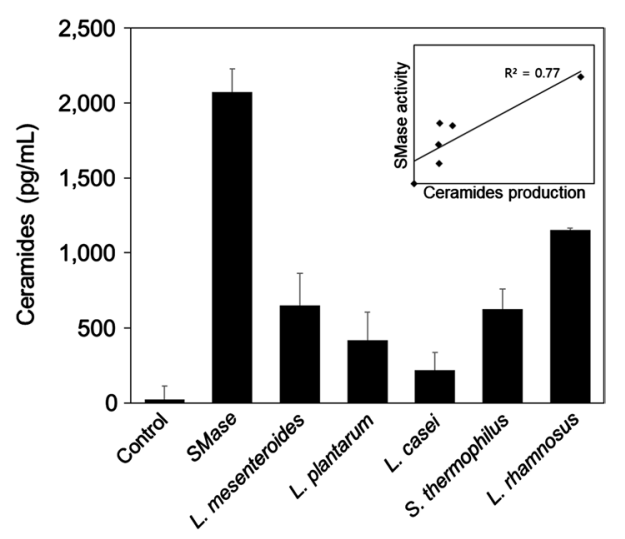

Fig. 3 Enhanced ceramides production by the lysates from $L$. rhamnosus IDCC 3201. Produced ceramides concentration was measured using an ELISA kit. The contents of ceramides were as follows: control, $21.4 \mathrm{pg} / \mathrm{mL}$; SMase $(10 \mu \mathrm{g} / \mathrm{mL}), 2189.2 \mathrm{pg} / \mathrm{mL} ; \mathrm{L}$. mesenteroides, 651.1 pg/mL; L. plantarum, 417.9 pg/mL; L. casei, $216.8 \mathrm{pg} / \mathrm{mL}$; S. thermophilus, $626.2 \mathrm{pg} / \mathrm{mL}$; L. rhamnosus $1153.5 \mathrm{pg} /$ $\mathrm{mL}$. The data represent means \pm standard deviations from triplicate experiments
Here, ceramides production was investigated whether enhanced SMase activity by L. rhamnosus IDCC 3201 affects ceramides production in HEK cells. Prior to investigating the impact of lysates of $L$. rahmonsus IDCC 3201, cytotoxicity of HEK by the lysates was assessed. As a result, no significant reduction of cell proliferation was observed at cell density, ranging from $1 \times 10^{7}$ to $5 \times 10^{8} \mathrm{CFU} / \mathrm{mL}$ for $24 \mathrm{~h}$ (Fig. 2). Furthermore, to substantiate that increased SMase activity is responsible for enhanced ceramides production, lysates of $L$. rhamnosus IDCC 3201 and lactic acid bacteria tested in Fig. 1 were treated in HEK cells. Overall, increased ceramides production is correlated with increased SMase activity $\left(R^{2}=0.77\right.$, Fig. 3$)$ to some degree. Ceramides concentration by $L$. rhamnosus IDCC 3201 was $1.7-5.2$ times higher than those by other lactic acid bacteria. More specifically, $1153.5 \pm 334.5 \mathrm{pg} / \mathrm{mL}$ of ceramides was measured in HEKs treated by L. rhamnosus IDCC 3201 , while $651.1 \pm 72.9,417.9 \pm 55.8,216.8 \pm 69.3$, and $626.2 \pm 216.5 \mathrm{pg} / \mathrm{mL}$ of ceramides were measured in HEKs by $L$. mesenteroides, L. plantarum, L. casei, and S. thermophilus, respectively (Fig. 3). The observation that HEK cells treated with the lysates of L. rhamnosus showed higher production of ceramides, might be due to the induction of higher SMase activity (Fig. 1). To validate this notion, real-time PCR was performed to analyze the expression levels of SMPD3, CERS1 and CERS2 responsible for ceramides synthesis: SMPD3 coding for sphingomyelin phosphodiesterase 3; CERS1 for ceramide synthase 1; CERS 2 for ceramide synthase 1. In results, the expression level of SMPD3 was significantly increased in response to L. rhamnosus IDCC 3201, but not in $L$. plantarum. More specifically, each strain exhibited $35 \%$ and $11.4 \%$ increase of SMPD3 expression, respectively
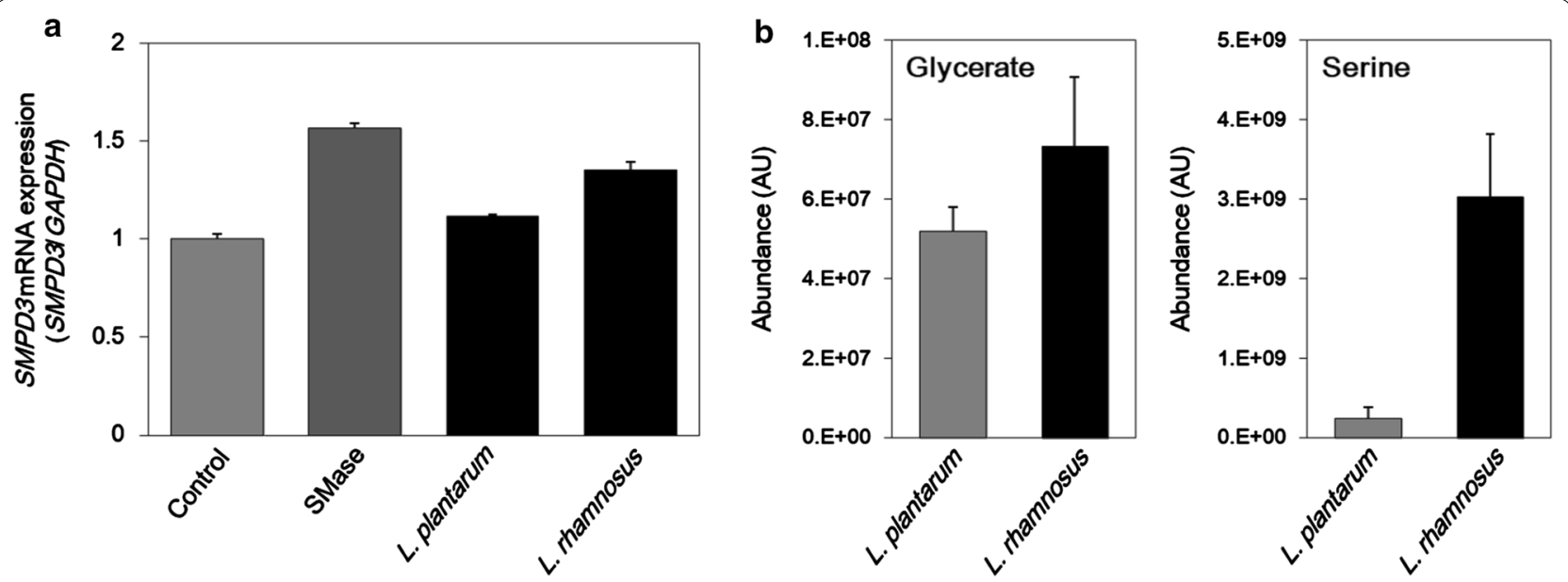

Fig. 4 RT-PCR and metabolome analysis. a Enhanced SMPD3 expression level in HEK treated by the lysates of L. plantarum and L. rhamnosus 3201. b Increased abundance of glycerate and serine in L. rhamnosus IDCC 3201 in comparison with L. plantarum strain 
(Fig. 4a). Based on the results, it was concluded that comparative higher production of cermides by $L$. rhamnosus IDCC 3201 was due to the up-regulation of SMPD3 rather than CERS1 or CERS2. As a conclusion, it is expected that lysates of L. rhamnosus IDCC 3201 contribute to the improvement of the skin barrier through the increased level of ceramides.

\section{Genome and metabolome analysis for elucidating a proposed mechanism}

To elucidate a mechanism of enhanced ceramides production by L. rhamnosus IDCC 3201, information of enzymes that can synthesize ceramides in microorganisms was collected (Table 1). Among 13 genes, L. rhamnosus IDCC 3201 harbored galc and $g b a$, which encodes for galactosylceramidase and glucosylceramidase, respectively. According to the gene annotation analysis using homology search, only $g b a$ gene was determined to be responsible for ceramides production from glucosylceramide, that is abundant in human keratinocytes [4]. Previous studies reported that the lack of glucosylceramidase encoded by the $g b a$ gene disturbed ceramides production and caused the failure of formation of competent skin barrier [9]. Thus, the increased ceramides in HEK cells was due to the expression of glucosylceramidase or increased SMase activity in the lysates of $L$. rhamonsus IDCC 3201. In order to specify the possible effects of lysate components on the enhanced ceramides production, we analyzed the metabolites present in the culture supernatants of L. rhamnosus and L. plantarum using GC-TOF mass spectroscopy. As shown in Fig. 4b, we found that abundances of glycerate and serine increased in the L. rhamnosus culture compared to L. plantarum. The first step of de novo synthesis of ceramides begins with the condensation of palmitate and serine, producing 3-keto-dihydrosphingosine. In this process, serine metabolism regulates ceramides and sphingolipid synthesis [27]. In addition, 3-phosphoglycerate, as one of the glycerate derivatives, is a precursor for the serine synthesis, supporting the notion that the increased glycerate and serine in the L. rhamnosus culture would induce the ceramides synthesis [28]. Finally, the reasons of enhanced ceramides production can be summarized by 1 ) indirect effect of components of the lysates (i.e., glycerate and serine) and 2) direct effect of glucosylceramidase on cermides production (Fig. 5).

\section{Safety evaluation of $L$. rhamnosus IDCC 3201}

Genome analysis of L. rhamnosus IDCC 3201 indicated that the size of the genome was approximately 3.05 Mbps

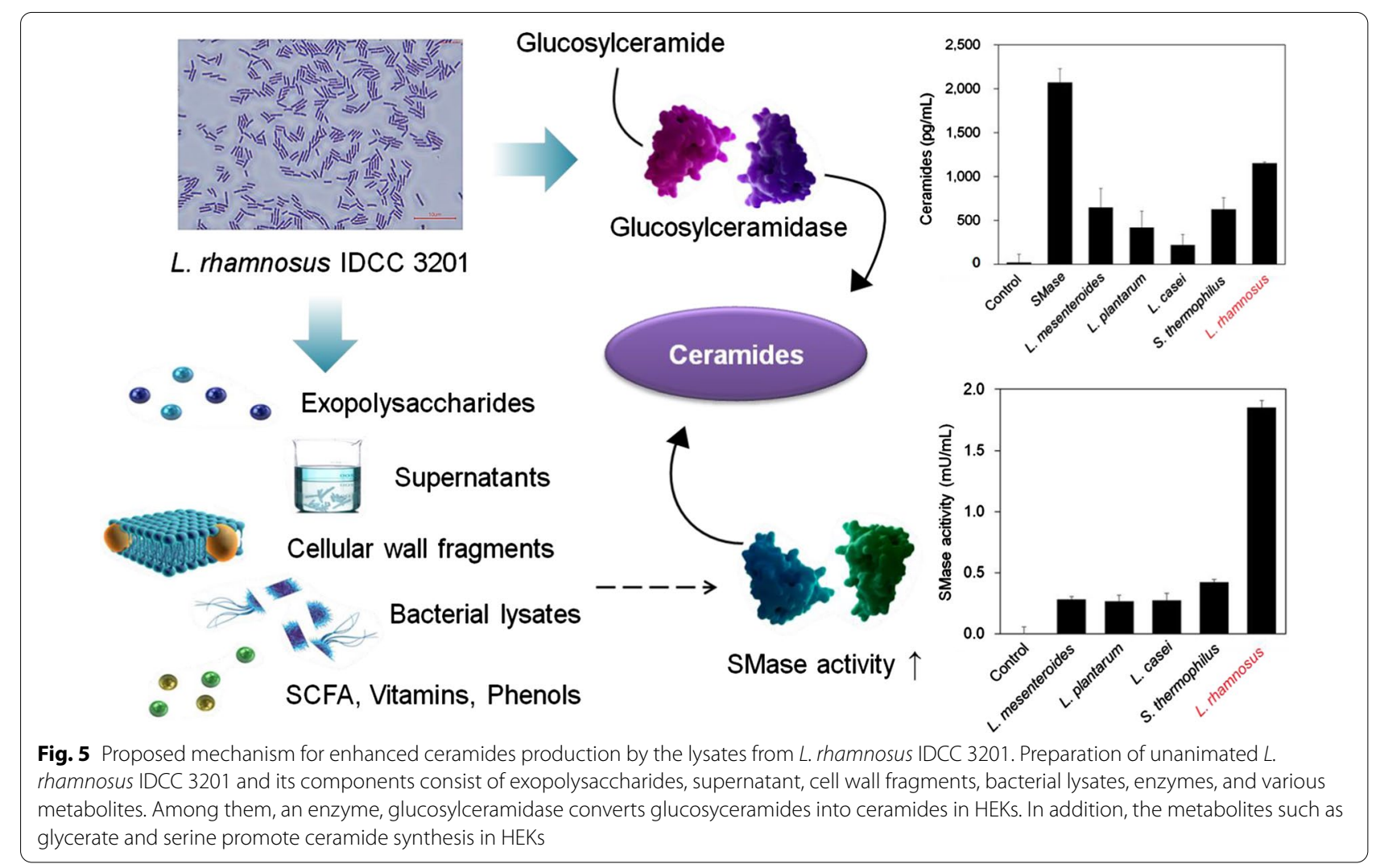




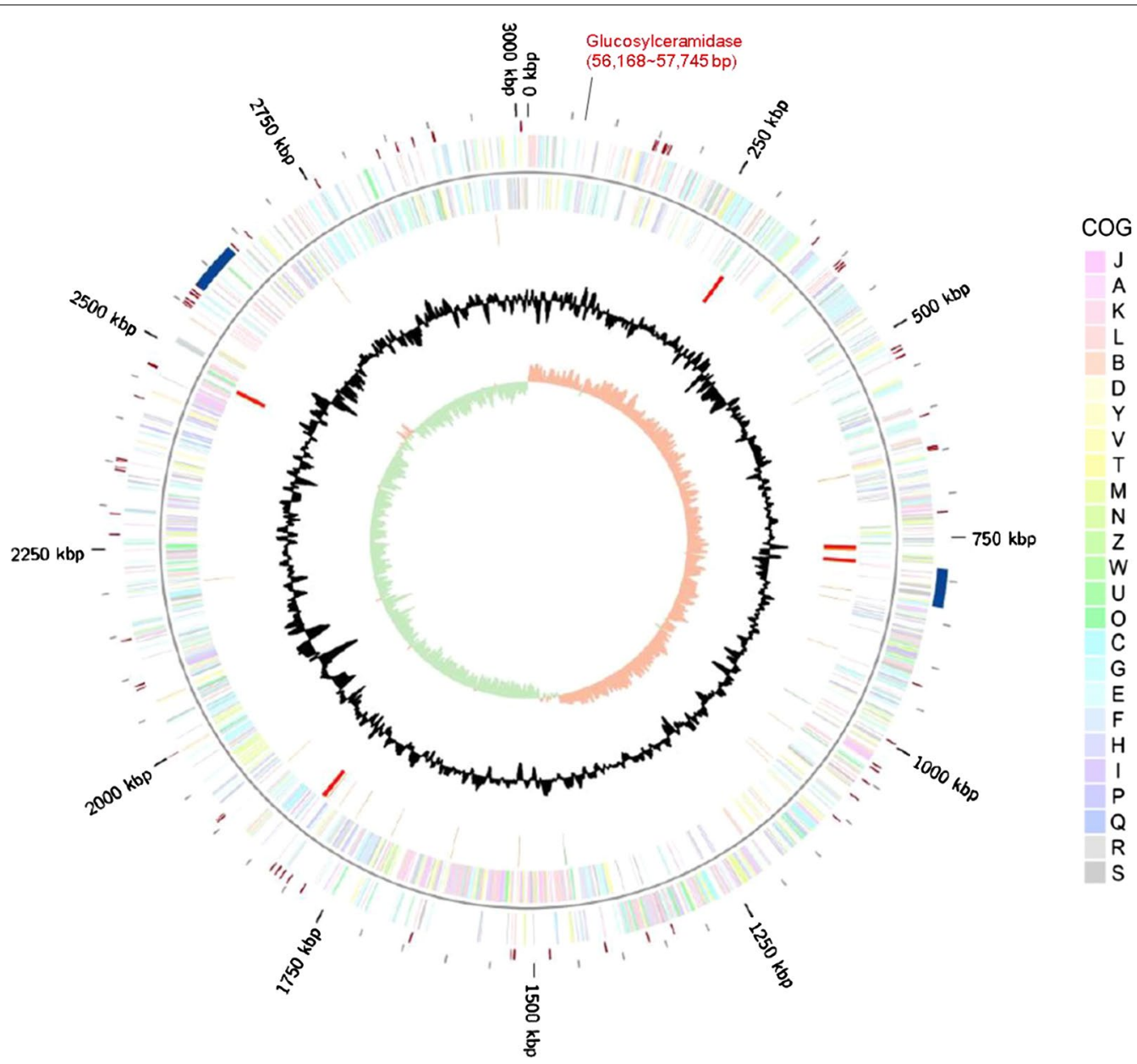

Fig. 6 Circular map of the chromosome of L. rhamnosus IDCC 3201. The whole genome was used for searching the genes responsible for ceramides production, antibiotic resistance, virulence, mobile elements, and prophage regions. Marked characteristics are shown from outside to the center, mobile elements (transposases, dark red; intact prophage region, dark blue), CDS on forward strand, CDS on reverse strand, RNA genes (tRNAs orange; rRNAs, red; other RNAs, green), GC content (black) and GC skew (light green/orange)

with a GC content of $46.71 \%$ and 2,821 functional genes (Additional file 1: Tables S1 and S2) (Fig. 6). Furthermore, L. rhamnosus IDCC 3201 does not have any toxigenic gene or virulence factor. According to the MIC tests, $L$. rhamnosus IDCC 3201 was susceptible to all of the antibiotics except for gentamycin and kanamycin (Table 3).

Table3 MICs of L. rhamnosus IDCC 3201 against a variety of antibiotics

\begin{tabular}{|c|c|c|c|c|c|c|c|c|c|}
\hline & AMP & VAN & GEN & KAN & STR & ERY & CLI & TET & $\mathrm{CHL}$ \\
\hline $\begin{array}{l}\text { Cut-off value } \\
(\mu \mathrm{g} / \mathrm{mL})^{\mathrm{a}}\end{array}$ & 4 & $n . r$ & 16 & 64 & 32 & 1 & 4 & 8 & 4 \\
\hline $\begin{array}{l}\text { L. rhamnosus } \\
\text { IDCC } 3201\end{array}$ & $1 / S^{b}$ & 512-1024 & $32 / R^{c}$ & $128 / \mathrm{R}$ & $32 / 5$ & $<0.125 / \mathrm{S}$ & $<0.125 / S$ & $0.25 / \mathrm{S}$ & $<0.125 / \mathrm{S}$ \\
\hline
\end{tabular}

n.r. not required

AMP ampicillin, VAN vancomycin, GEN gentamicin, KAN kanamycin, STR streptomycin, ERY erythromycin, $C L I$ clindamycin, $T E T$ tetracycline, $C H L$ chloramphenicol

${ }^{a}$ EFSA (European Food Safety Authority), 2018. EFSA Journal, 16(3), 5206

${ }^{b}$ S: Susceptible

${ }^{\mathrm{C}} \mathrm{R}$ : Resistant 
These resistances should be intrinsic and not be transferable to skin microbes due to the absence of antibiotic resistance gene. Typically, many Lactobacillus species are shown to be tolerant to aminoglycoside antibiotics intrinsically due to the absence of cytochrome-mediated transport [29, 30]. In conclusion, L. rhamnosus IDCC 3201 strain is regarded as safe for its use as cosmetic materials.

\begin{abstract}
Abbreviations
GCase: Glucosylceramidase; SMase: Sphingomyelinase; AD: Atopic dermatitis; MRS: De Man, Rogosa and Sharpe; CFU: Colony forming unit; PBS: Phosphate buffered solution; HEK: Human epidermal keratinocyte; MTT: 3-(4,5-Dimethylthiazol-2-yl)-2,5-diphenyltetrazolium bromide; DMSO: Dimethyl sulfoxide; ELISA: Enzyme-linked immunosorbent assay; MIC: Minimal inhibitory concentration; EFSA: European food safety authority; mU: Milli unit; Mbp: Mega base pair.
\end{abstract}

\section{Supplementary Information}

The online version contains supplementary material available at https://doi. org/10.1186/s13765-021-00620-7.

Additional file 1: Table S1. Statistical parameters of genome of $L$. rham nosus IDCC 3201. Table S2. Functional genes of L. rhamnosus IDCC 3201.

\section{Acknowledgements}

The article-processing charge is supported by Ildong Bioscience.

\section{Authors' contributions}

JY designed the research, supervised the project, and finalized the manuscript. MSK, ML, and WS designed the research and contributed to the writing of the manuscript. MSK, ML, HO, GK, OB, and MS performed the experiments and analyzed the data. ML, MS and YHJ generated and provided analytical tools. $\mathrm{YHJ}$ aided in interpreting the results and provided critical revision. All authors read and approved the final manuscript.

\section{Funding}

This work was supported by Gyeonggido Business \& Science Accelerator (GBSA).

\section{Availability of data and materials}

Supplementary material is followed.

\section{Declarations}

\section{Competing interests}

The authors have no financial conflicts of interest to declare.

\section{Author details}

${ }^{1}$ INTERCARE, 1 Naeyuri 1-gil, Cheonan-si, Chungcheongnam-do 31027, Republic of Korea. ${ }^{2}$ lldong Bioscience, 17 Poseunggongdan-ro, Pyeongtaek-si, Gyeonggi-do 17957, Republic of Korea. ${ }^{3} \mid l d o n g$ Pharmaceutical, 2 Baumoe-ro 27-gil, Seoul 06752, Republic of Korea. ${ }^{4}$ School of Food Science and Biotechnology, Kyungpook National University, Daegu 41566, Republic of Korea. ${ }^{5}$ Department of Agricultural Biotechnology and Research Institute of Agriculture and Life Science, Seoul National University, Seoul 00826, Republic of Korea. ${ }^{6}$ Institute of Fermentation Biotechnology, Kyungpook National University, Daegu 41566, Republic of Korea.

Received: 1 March 2021 Accepted: 24 June 2021

Published online: 02 July 2021

\section{References}

1. Araviiskaia E, Berardesca E, Bieber T, Gontijo G, Sanchez Viera G, Marrot L et al (2019) The impact of airborne pollution on skin. J Eur Acad Dermatol Venereol 33(8):1496-1505

2. Meckfessel MH, Staci Brandt S (2019) The structure, function, and importance of ceramides in skin and their use as therapeutic agents in skin-care products. J Am Acad Dermatol 71(1):177-184

3. Di Marzio L, Cinque B, Cupelli F, De Simone C, Cifone MG, Giuliani M (2008) Increase of skin-ceramide levels in aged subjects following a short-term topical application of bacterial sphingomyelinase from Streptococcus thermophilus. Int I Immunopathol Pharmacol 21(1):137-143

4. Choi MJ, Maibach HI (2005) Role of ceramides in barrier function of healthy and diseased skin. Am J Clin Dermatol 6(4):215-223

5. Li Q, Fang H, Dang E, Wang G (2020) The role of ceramides in skin homeostasis and inflammatory skin diseases. J Dermatol Sci 97(1):2-8

6. Hamanaka S, Hara M, Nishio H, Otsuka F, Suzuki A, Uchida Y (2002) Human epidermal glucosylceramides are major precursors of stratum corneum ceramides. J Invest Dermatol 119(2):416-423

7. Imokawa G, Akasaki S, Minematsu Y, Kaway M (1989) Importance of intercellular lipids in water retention properties of the stratum corneum: induction and recovery study of surfactant dry skin. Arch Dermatol Res 281(1):45-51

8. Mizutani Y, Misutake S, Tsuji K, Kihara A, Igarashi Y (2009) Ceramide biosynthesis in keratinocyte and its role in skin function. Biochimie 91(6):784-790

9. Rabionet M, Gorgas K (1841) Sandhoff R (2014) Ceramide synthesis in the epidermis. Biochim Biophys Acta 3:422-434

10. Ozen M, Dinleyici EC (2015) The history of probiotics: the untold story. Benef Microbes 6(2):159-165

11. Sivamaruthi BS, Kesika P, Chaiyasut C (2018) A review on anti-aging properties of probiotics. Int J App Pharm 10(5):23-27

12. Di Marzio L, Cinque B, De Simone C, Cifone MG (1999) Effect of the lactic acid bacterium Streptococcus thermophilus on ceramide levels in human keratinocytes in vitro and stratum corneum in vivo. J Invest Dermatol 113(1):98-106

13. Di Marzio L, Cinque B, Cupelli F, De Simone C, Cifone MG, Giulianin M (2008) Skin-ceramide levels in aged subjects following a short-term topical application of bacterial sphingomyelinase from Streptococcus Thermophilus. Int J Immunopathol Pharmacol 21(1):137-143

14. Jung YO, Jeong H, Cho Y, Lee EO, Jang HW, Kim J et al (2019) Lysates of a probiotic, Lactobacillus rhamnosus, can improve skin barrier function in a reconstructed human epidermis model. Int J Mol Sci 20(17):4289

15. Lee S-H, Yoon J-M, Kim Y-H, Jeong D-G, Park S, Kang D-J (2016) Therapeutic effect of tyndallized Lactobacillus rhamnosus IDCC 3201 on atopic dermatitis mediated by down-regulation of immunoglobulin $\mathrm{E}$ in NC/Nga mice. Microbiol Immunol 60(7):468-476

16. Ban O-H, Oh S, Park C, Bang WY, Lee BS, Yang S-Y et al (2020) Safety assessment of Streptococcus thermophilus IDCC 2201 used for product manufacturing in Korea. Food Sci Nutr 8(11):6269-6274

17. Hannun YA, Obeid LM (2008) Principles of bioactive lipid signalling: lessons from sphingolipids. Nat Rev Mol Cell Biol 9(2):139-150

18. Eddy SR (2011) Accelerated profile HMM searches. PLoS Comput Biol 7(10):e1002195

19. Chen L, Yang J, Yu J, Yao Z, Sun L, Shen Y et al (2005) VFDB: a reference database for bacterial virulence factors. Nucl Acid Res 33:D325-D328

20. Zankari $\mathrm{E}$, Hasman $\mathrm{H}$, Cosentino S, Vestergaard M, Rasmussen S, Lund O et al (2012) Identification of acquired antimicrobial resistance genes. J Antimicrob Chemother 67(11):2640-2644

21. Arndt D, Grant JR, Marcu A, Sajed T, Pon A, Liang Y et al (2016) PHASTER: a better, faster version of the PHAST phage search tool. Nucl Acid Res 44(W1):W16-W21

22. Wiegand I, Hilpert K, Hancock REW (2008) Agar and broth dilution methods to determine the minimal inhibitory concentration (MIC) of antimicrobial substances. Nat Protoc 3(2):163-175

23. Rychen G, Aquilina G, Azimonti G et al (2018) Guidance on the characterization of microorganisms used as feed additives or as production organisms. EFSA J 16(3):05206

24. Kornhuber J, Rhein C, Müller CP, Mühle C (2015) Secretory sphingomyelinase in health and disease. Bio Chem 396(6-7):707-736 
25. Jensen JM, Fo"lster-Holst R, Baranowsky A, Schunck M, Winoto-Morbach S, Neumann C, et al (2004) Impaired sphingomyelinase activity and epidermal differentiation in atopic dermatitis. J Invest Dermatol 122(6):1423-1431

26. Jonca N (2019) Ceramides metabolism and impaired epidermal barrier in cutaneous diseases and skin aging: focus on the role of the enzyme PNPLA1 in the synthesis of $\omega$-O-acylceramides and its pathophysiological involvement in some forms of congenital ichthyoses. OCL 26:17

27. Gao X, Lee K, Reid MA, Sanderson SM, Qiu C, Li S et al (2018) Serine availability influences mitochondrial dynamics and function through lipid metabolism. Cell Rep 22(13):3507-3520

28. Okamura E, Hirai MY (2017) Novel regulatory mechanism of serine biosynthesis associated with 3-phosphoglycerate dehydrogenase in Arabidopsis thaliana. Sci Rep 7(1):1-14
29. Abriouel H, Casado Muñoz MC, Lerma LL, Montoro BP, Bokelmann W, Pichner $R$ et al (2015) New insights in antibiotic resistance of Lactobacillus species from fermented foods. Food Res Int 78:465-481

30. Ammor MS, Flórez AB, Mayo B (2007) Antibiotic resistance in nonenterococcal lactic acid bacteria and bifidobacteria. Food Microbiol 24(6):559-570

\section{Publisher's Note}

Springer Nature remains neutral with regard to jurisdictional claims in published maps and institutional affiliations.

\section{Submit your manuscript to a SpringerOpen ${ }^{\circ}$ journal and benefit from:}

- Convenient online submission

- Rigorous peer review

- Open access: articles freely available online

- High visibility within the field

- Retaining the copyright to your article

Submit your next manuscript at $\boldsymbol{\nabla}$ springeropen.com 